\title{
Effectiveness and Safety of Basal Insulin Therapy in Type 2 Diabetes Mellitus Patients With or Without Metformin Observed in a National Cohort in China
}

\section{Puhong Zhang}

The George Institute for Global Health at Peking University Health Science Center https://orcid.org/0000-0003-4610-9848

\section{Heng Zhang}

The George Institute for Global Health at Peking University Health Science Center

\section{Yingying Luo}

Department of Endocrinology and Metabolism, Peking University People's Hospital

\section{Dongshan Zhu}

The George Institute for Global Health at Peking University Health Science Center

\section{Xian Li}

The George Institute for Global Health at Peking University Health Science Center

\section{Jiachao Ji}

The George Institute for Global Health at Peking University Health Science Center

\section{Minyuan Chen}

The George Institute for Global Health at Peking University Health Science Center

\section{Du Wang}

The George Institute for Global Health at Peking University Health Science Center

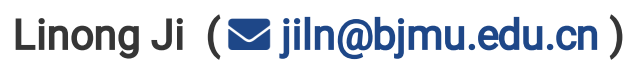

The George Institute for Global Health at Peking University Health Science Center

\section{Research article}

\section{Keywords:}

Posted Date: February 12th, 2021

DOl: https://doi.org/10.21203/rs.3.rs-228534/v1

License: (c) (1) This work is licensed under a Creative Commons Attribution 4.0 International License. Read Full License 


\section{Abstract \\ Background.}

Though many randomized control trials had examined the effectiveness and safety of taking insulin therapy with or without metformin, there are limited real-world data, especially among Chinese type 2 diabetes patients initiating basal insulin (BI) with uncontrolled hyperglycemia by oral agents. This study was designed to assess the effectiveness and safety of BI therapy combined with or without metformin in a real-world national cohort study.

\section{Methods.}

Patients with type 2 diabetes mellitus who initiated BI treatment due to uncontrolled hyperglycemia $(\mathrm{HbA} 1 \mathrm{c} \geq 7 \%$ ) by oral antidiabetic drugs (OADs) were recruited in Chinese real-world settings between 2011 and 2013. A total of 12,358 patients initiated BI without bolus insulin and completed a 6-month follow-up were selected as the study population and divided into BI with metformin or BI without metformin group based on whether metformin was simultaneously prescribed or not at baseline. Propensity score adjustment was used to balance baseline covariates between two groups. A subanalysis was also conducted among 8,086 patients who kept baseline treatment regimen during the follow-up. Outcomes were HbA1c, hypoglycemia, weight gain and insulin dose in two groups.

\section{Results.}

$53.6 \%(6,621$ out of 12,358$)$ patients initiated BI therapy concomitant with metformin. After propensity score adjustment, multivariate regression analysis controlled with number of OADs, total insulin dose, physical activity and diet consumption showed that BI with metformin group had a slightly higher control rate of $\mathrm{HbA} 1 \mathrm{c}<7.0 \%(39.9 \%$ vs. $36.4 \%, \mathrm{P}=0.0011)$ at 6 -month follow-up, and lower dose increment from baseline to 6 -month $(0.0064$ vs. $0.0068 \mathrm{U} /$ day $/ \mathrm{kg}, \mathrm{P}=0.0035)$. The sub-analysis with patients remained at same BI therapy further showed that BI with metformin group had higher $\mathrm{HbA} 1 \mathrm{c}$ control rate $(47.9 \% \mathrm{vs}$. $41.9 \%, P=0.0001)$, less weight gain $(-0.12$ vs $0.15 \mathrm{~kg} \mathrm{P}=0.0013)$, and lower dose increment during 6month follow-up (0.0033 vs. $0.0037 \mathrm{U} /$ day $/ \mathrm{kg}, \mathrm{P}=0.0073$ ) when compared with $\mathrm{BI}$ without metformin group.

\section{Conclusion.}

In alliance with current guidelines, the real-world findings also support the insulin initiation together with metformin. Continuous patients' education and clinicians training are needed to improve the use of metformin when initiating BI treatment. 


\section{Background}

With estimated 113.9 million people in China and 420 million globally affected, diabetes continues to be one of the most common non-communicable diseases and public health priorities worldwide $(1,2)$. Type 2 diabetes mellitus (T2DM) is the main category of diabetes in adults, which is characterized by steady deterioration of glycemic control due to progressive $\beta$-cell dysfunction of insulin secretion frequently on the background of insulin resistance. The objective of T2DM therapy is to safely achieve and maintain glycemic control in order to reduce the risk of microvascular and macrovascular complications, and in the long run, diabetes related mortality. However, as achieving a near-normal glycemic control target becomes increasingly difficult due to disease progression, most patients ultimately require insulin treatment (3-5).

Although timely addition of insulin to oral therapy for T2DM has been recommended to prevent complications by early institution of strict glycemic control and $\beta$-cell protection (3), a substantial proportion of patients with suboptimal glucose control tend to delay insulin therapy due to fear of hypoglycemia, along with a reluctance to start injections and gain weight (6), which may further influence adherence to insulin therapy (7). Metformin as first line therapy for T2DM is recommended to be continued after initiating insulin (usually basal insulin (BI)) by current guidelines (8-10) since the regimen of insulin plus concomitant metformin was shown to improve glycemic control, reduce hypoglycemia incidence and weight gain as well as total insulin dose when compared with insulin monotherapy (11-14). In addition, this combination regimen was reported to reduce the risk of death and macrovascular events compared with insulin monotherapy in the long run (15). Though many randomized control trials had examined the effectiveness and safety of taking insulin therapy with or without metformin(16) (14) (17) (18), there are limited real-world data, especially among Chinese T2DM initiating $\mathrm{BI}$ due to uncontrolled hyperglycemia by oral antidiabetic drugs (OADs).

Therefore, based on the data of the Observational Registry for BI Treatment (ORBIT) study (19), we analyzed the real-world practice combination of metformin with BI initiation, and compare the effectiveness and safety between Bl users with and without metformin in a Chinese real-world setting.

\section{Methods}

\section{Study design and participants}

ORBIT was a real-world cohort study conducted from 2011 to 2013. Patients in age of 18-80 years and initiating $\mathrm{BI}$ treatment due to inadequately $\mathrm{HbA} 1 \mathrm{c}$ control (HbA1c $\geq 7 \%)$ by OADs were recruited from 209 hospitals in Mainland China and followed up for 6 months. Details on the study design were reported previously (19).The ORBIT protocol was approved by the Institutional Review Board (IRB) of Peking University and, when necessary, by local IRBs. Written informed consents were obtained from all patients.

\section{Data collection and data used for this study}


Study data were collected through interview at baseline (visit 1), 3 months (visit 2) and end of the study (6-month, visit 3), including demographics (gender, age, education, patient recruitment settings, current residence and medical insurance), clinical characteristics (diabetes duration, OADs treatment duration before BI therapy), standard physical examination (body weight, height and blood pressure), number and types of OADs before and after initiating $\mathrm{BI}$, hypoglycemia, lifestyle involving food intake and physical activity, and laboratory test of fasting plasma glucose (FPG) and HbA1c at both visit 1 (v1) and visit 3 (v3). Only patients who initiated $\mathrm{BI}$ alone (without prandial insulin) and completed the 6-month follow-up were included in this study.

\section{Grouping method and outcomes}

The study population were divided into BI with metformin or BI without metformin group based on whether BI was simultaneously combined with metformin or not at baseline. A sub-analysis was also conducted among patients who remained at baseline treatment of $\mathrm{BI}$ with or without metformin throughout the 6 months follow-up. The primary outcomes of the study were control rate of $\mathrm{HbA} 1 \mathrm{c}$ (< $7.0 \%$ or $\leq 6.5 \%$ ) and incidence of hypoglycemia including severe hypoglycemia, documented symptomatic, asymptomatic, probable symptomatic or pseudo-hypoglycemia according to the American Diabetes Association recommendations (20) at v3. Secondary outcomes were mean levels of HbA1c, FPG, weight and insulin dose; control rate of FPG (<7.0 mmol/L) at v3; and mean changes of HbA1c, FPG, weight and insulin dose at v3 compared with v1.

\section{Statistical analyses}

Multivariate analysis based on propensity score adjustment was used to compare the outcomes between two groups. Descriptive statistics, including means \pm SD for continuous variables and $n(\%)$ for categorical variables, were used to present the demographic and clinical characteristics of patients before and after propensity score adjustment respectively. Levels in HbA1c, FPG, body weight and insulin dose were analyzed by using propensity score-adjusted multivariate linear regression models. Rates of hypoglycemia, control rates of HbA1c and FPG were analyzed by using propensity score-adjusted multivariate logistic regression models.

A propensity score was calculated by using logistic regression analysis in this nonrandomized sample; it defines the conditional probability for an individual to be exposed to BI with metformin, on the basis of baseline covariates. The propensity scores were used to adjust for differences of baseline covariates between $\mathrm{BI}$ with metformin group and $\mathrm{BI}$ without metformin group, including regions, current residence, hospital level, medical insurance (actual proportion of out of pocket), gender, age, body mass index (BMI), duration of diabetes and OAD treatment, HbA1c and FPG levels, hypoglycemia events at baseline, diabetes complication, number and type of OADs prior to BI therapy, and BI types used at baseline (21, 22). Before and after propensity score adjustment, Pearson's chi square test and unpaired t-test were used to analyze differences in proportions and continuous variables of baseline values, respectively; balance in variables was indicated when $P$ values $>0.05$ after propensity score adjustment. Process confounding factors which could change during the follow-up were also analyzed, including number of OADs, total 
insulin dose, physical activity and diet consumption at visit 3 . In regression analyses of outcomes, baseline covariates were adjusted by propensity scores and process confounding factors were controlled in regression models.

All reported $P$ values were two sided, with $P$ values $<0.05$ considered to be statistically significant. Statistical analyses were performed with Statistical Analysis System, version 9.4 (SAS Institute, Cary, NC, USA).

\section{Results}

Among all the recruited 18,995 participants at baseline, 12,358 (65.1\%) patients who initiated BI only therapy and completed 6 months follow-up were included in the analysis. Figure 1 shows the patients selection procedure.

Patients' characteristics and metformin use

Overall of the 12,358 patients, males accounted for $53.1 \%$ and the mean age was 55.5 years. Patients had a mean diabetes duration of 6.2 years and OAD treatment duration of 5.5 years, BMI of $24.8 \mathrm{~kg} / \mathrm{m}^{2}$, $\mathrm{HbA} 1 \mathrm{c}$ of $9.3 \%$, and FPG of $11.2 \mathrm{mmol} / \mathrm{L}$.

Before propensity score adjustments, baseline variables including age, recruitment settings (inpatient ward or outpatient clinic), current residence (urban or rural area), out of pocket ratio, BMI, diabetes and OAD treatment duration before BI therapy, systolic and diastolic blood pressure, $\mathrm{HbA} 1 \mathrm{c}$ level at baseline, episodes of hypoglycemia, microvascular and macrovascular complications, number and types of OADs (metformin, a-glycosidase inhibitors and glinides) before BI therapy, and types of basal insulin at BI initiation were significantly different between $\mathrm{BI}$ with metformin and BI without metformin two groups. Balance of baseline characteristics between the two groups was achieved among most variables after propensity score adjustments except for metformin before BI therapy and episodes of hypoglycemia. (Table 1) 
Table 1

Baseline characteristics of patients with basal insulin therapy before and after propensity score adjustment

\begin{tabular}{|c|c|c|c|c|}
\hline & \multicolumn{2}{|c|}{$\begin{array}{l}\text { Before propensity score } \\
\text { adjustment }\end{array}$} & \multicolumn{2}{|c|}{$\begin{array}{l}\text { After propensity score } \\
\text { adjustment }\end{array}$} \\
\hline & $\begin{array}{l}\text { BI with } \\
\text { metformin }\end{array}$ & $\begin{array}{l}\text { Bl without } \\
\text { metformin }\end{array}$ & $\begin{array}{l}\text { BI with } \\
\text { metformin }\end{array}$ & $\begin{array}{l}\text { Bl without } \\
\text { metformin }\end{array}$ \\
\hline & $(N=6621)$ & $(\mathrm{N}=5737)$ & $(N=6621)$ & $(N=5737)$ \\
\hline \multicolumn{5}{|l|}{ Demographics } \\
\hline Male (\%) & 52.8 & 53.4 & 53.1 & 53.1 \\
\hline Age (years, Mean \pm SD) & $54.2 \pm 9.9 * \star$ & $56.8 \pm 10.4$ & $55.5 \pm 10.9$ & $55.4 \pm 11.0$ \\
\hline \multicolumn{5}{|l|}{ Education (\%) } \\
\hline Primary school or illiterate & 24.9 & 26.0 & 25.2 & 25.7 \\
\hline Junior high school & 30.8 & 29.2 & 30.3 & 29.8 \\
\hline Senior high school & 25.6 & 26.2 & 25.9 & 25.9 \\
\hline Junior college & 10.8 & 11.5 & 11.2 & 11.1 \\
\hline Bachelor's degree or higher & 7.9 & 7.1 & 7.4 & 7.5 \\
\hline \multicolumn{5}{|l|}{ Hospital level (\%) } \\
\hline Secondary hospitals & 49.8 & 50.4 & 50.1 & 50.1 \\
\hline Tertiary hospitals & 50.2 & 49.6 & 49.9 & 49.9 \\
\hline \multicolumn{5}{|l|}{ Recruitment settings (\%) } \\
\hline Outpatient clinic & $54.5^{\star}$ & 51.2 & 53.0 & 53.0 \\
\hline Inpatient ward & 45.5 & 48.8 & 47.0 & 47.0 \\
\hline \multicolumn{5}{|l|}{ Current residence (\%) } \\
\hline Urban & $69.1^{\star *}$ & 73.2 & 71.1 & 71.1 \\
\hline Rural & 30.9 & 26.8 & 28.9 & 28.9 \\
\hline Out of pocket $(\%$, Mean \pm SD $)$ & $\begin{array}{l}42.5 \pm \\
28.1^{*}\end{array}$ & $40.8 \pm 27.2$ & $41.7 \pm 30.3$ & $41.7 \pm 30.7$ \\
\hline \multicolumn{5}{|l|}{ Clinical } \\
\hline BMI $\left(\mathrm{kg} / \mathrm{m}^{2}\right.$, Mean $\left.\pm \mathrm{SD}\right)$ & $25.4 \pm 3.3^{\star \star}$ & $24.2 \pm 3.2$ & $24.9 \pm 3.5$ & $24.8 \pm 3.5$ \\
\hline $\begin{array}{l}\text { Duration of diabetes (years, Mean } \pm \\
\text { SD) }\end{array}$ & $6.2 \pm 5.0 *$ & $6.5 \pm 5.3$ & $6.4 \pm 5.6$ & $6.4 \pm 5.7$ \\
\hline
\end{tabular}




\begin{tabular}{|c|c|c|c|c|}
\hline \multirow[b]{2}{*}{$\begin{array}{l}\text { Duration of OAD treatment (years, } \\
\text { Mean } \pm \text { SD) }\end{array}$} & \multicolumn{2}{|c|}{$\begin{array}{l}\text { Before propensity score } \\
\text { adjustment }\end{array}$} & \multicolumn{2}{|c|}{$\begin{array}{l}\text { After propensity score } \\
\text { adjustment }\end{array}$} \\
\hline & $5.5 \pm 4.9 *$ & $5.7 \pm 5.0$ & $5.6 \pm 5.4$ & $5.6 \pm 5.5$ \\
\hline SBP $(m m H g$, Mean \pm SD $)$ & $\begin{array}{l}130.1 \pm \\
15.4^{\star}\end{array}$ & $129.3 \pm 15.6$ & $\begin{array}{l}129.9 \pm \\
17.0\end{array}$ & $129.4 \pm 17.2$ \\
\hline DBP $(m m H g$, Mean \pm SD $)$ & $79.9 \pm 9.2^{\star \star}$ & $79.1 \pm 9.2$ & $79.4 \pm 10.0$ & $79.6 \pm 10.2$ \\
\hline $\mathrm{HbA} 1 \mathrm{c}$ at baseline $(\%$, Mean \pm SD $)$ & $9.4 \pm 1.8^{\star \star}$ & $9.2 \pm 1.8$ & $9.3 \pm 2.0$ & $9.3 \pm 2.0$ \\
\hline $\begin{array}{l}\text { FPG at baseline }(\mathrm{mmol} / \mathrm{L}, \text { Mean } \pm \\
\text { SD) }(\text { missing }=616)\end{array}$ & $11.4 \pm 3.5$ & $11.0 \pm 3.6$ & $11.2 \pm 3.8$ & $11.2 \pm 3.9$ \\
\hline \multicolumn{5}{|l|}{ General hypoglycemia at baseline } \\
\hline Percentage (\%) & 5.7 & 5.8 & 5.7 & 5.8 \\
\hline $\begin{array}{l}\text { Episodes (times } / 100 \text { person-year, } \\
\text { Mean } \pm \text { SD) }\end{array}$ & $1.7 \pm 9.6^{*}$ & $1.6 \pm 9.9$ & $1.7 \pm 1.4^{\star}$ & $1.6 \pm 1.4$ \\
\hline \multicolumn{5}{|l|}{ Complication at baseline (\%) } \\
\hline Macrovascular & $13.5^{\star}$ & 15.6 & 14.4 & 14.4 \\
\hline Microvascular & $24.6^{\star}$ & 27.5 & 25.9 & 25.9 \\
\hline \multicolumn{5}{|l|}{ Pre-insulin OAD type ${ }^{a}(\%)$} \\
\hline Metformin & $87.8^{\star \star}$ & 39.1 & $96.0 *$ & 97.3 \\
\hline Sulfonylureas & 46.5 & 48.5 & 47.4 & 47.4 \\
\hline a-glycosidase inhibitors & $18.1^{\star \star}$ & 33.6 & 23.0 & 22.8 \\
\hline Glinides & $13.4^{\star \star}$ & 18.9 & 15.4 & 15.3 \\
\hline Thiazolidinediones & 5.7 & 6.4 & 6.0 & 6.0 \\
\hline Others & $8.7^{\star}$ & 9.8 & 9.2 & 9.2 \\
\hline \multicolumn{5}{|l|}{$\begin{array}{l}\text { Pre-insulin OAD number at baseline } \\
(\%)\end{array}$} \\
\hline 1 & $35.2^{\star \star}$ & 51.9 & 43.0 & 43.0 \\
\hline 2 & 50.9 & 40.9 & 48.1 & 48.2 \\
\hline$\geq 3$ & 13.9 & 7.1 & 8.9 & 8.8 \\
\hline \multicolumn{5}{|l|}{ BI type initiated at $\mathrm{v} 1$} \\
\hline Glargine & $69.6 * \star$ & 78.1 & 74.9 & 74.9 \\
\hline Detemir & 14.4 & 12.5 & 13.7 & 13.7 \\
\hline
\end{tabular}




\begin{tabular}{|c|c|c|c|c|}
\hline \multirow[b]{2}{*}{$\mathrm{NPH}$} & \multicolumn{2}{|c|}{$\begin{array}{l}\text { Before propensity score } \\
\text { adjustment }\end{array}$} & \multicolumn{2}{|c|}{$\begin{array}{l}\text { After propensity score } \\
\text { adjustment }\end{array}$} \\
\hline & 16.0 & 9.4 & 11.4 & 11.4 \\
\hline BI dose at v1 (U/day/kg) & $\begin{array}{l}0.17 \pm \\
0.071\end{array}$ & $0.18 \pm 0.069$ & $0.18 \pm 0.46$ & $0.18 \pm 0.47$ \\
\hline \multicolumn{5}{|c|}{$\begin{array}{l}\text { a Cumulative proportions might be over } 100 \text { percent as some patients might use two or more OADs. } \\
\star \star:<0.0001 ; *:<0.05 \text {. }\end{array}$} \\
\hline \multicolumn{5}{|c|}{$\begin{array}{l}\text { Abbreviation: BI, basal insulin; BMI, body mass index; OAD, oral antidiabetic drug; SBP, systolic blood } \\
\text { pressure; DBP, diastolic blood pressure; HbA1c, glycated hemoglobin; FPG, fasting plasma glucose; } \\
\text { NPH, Neutral Protamine Hagedorn. }\end{array}$} \\
\hline
\end{tabular}

Confounding factors after 6-month follow-up

Table 2 listed the comparison between patients initiating basal insulin with and without metformin for both full population analysis and sub-analysis, including number of OADs, insulin dose, diet and physical exercise status after 6-month follow-up at visit 3. In full and sub-populations, all the listed factors at visit 3 were significantly different between two groups. 
Table 2

Comparison of diabetes management between patients initiating basal insulin with and without metformin after 6-month follow-up at visit 3, (Mean \pm SD)

\begin{tabular}{|c|c|c|}
\hline & $\begin{array}{l}\text { Bl with } \\
\text { metformin }\end{array}$ & $\begin{array}{l}\text { Bl without } \\
\text { metformin }\end{array}$ \\
\hline Full population initiating $B I$ therapy $(N=12358)$ & $\begin{array}{l}N=6621 \\
(53.6 \%)\end{array}$ & $N=5737(46.4 \%)$ \\
\hline OAD number per day at visit 3 & $1.63 \pm 0.76$ & $1.19 \pm 0.74$ \\
\hline BI dose at visit3 (U/day/kg) & $0.16 \pm 0.12$ & $0.18 \pm 0.11$ \\
\hline Insulin dose at visit3 (U/kg/day) & $0.18 \pm 0.13$ & $0.20 \pm 0.13$ \\
\hline Staple food at visit3 (g) & $161.16 \pm 44.02$ & $156.15 \pm 42.69$ \\
\hline Vegetables at visit3 (g) & $188.08 \pm 66.49$ & $184.61 \pm 64.72$ \\
\hline Fruit at visit3 (g) & $100.17 \pm 42.76$ & $98.62 \pm 39.86$ \\
\hline Meat at visit3 (g) & $104.94 \pm 37.26$ & $103.09 \pm 35.21$ \\
\hline $\begin{array}{l}\text { Days of participating in }>30 \text { min physical activity in past } 7 \\
\text { days at visit3 }\end{array}$ & $5.98 \pm 1.99$ & $5.86 \pm 2.09$ \\
\hline $\begin{array}{l}\text { Days of participating in specific exercise session in past } 7 \\
\text { days at visit3 }\end{array}$ & $4.11 \pm 2.91$ & $4.00 \pm 2.90$ \\
\hline $\begin{array}{l}\text { Sub-population keeping BI therapy during 6-month follow-up } \\
(\mathrm{N}=8068)\end{array}$ & $\begin{array}{l}N=4269 \\
(52.9 \%)\end{array}$ & $N=3799(47.1 \%)$ \\
\hline OAD number per day at visit 3 & $1.75 \pm 0.65$ & $1.08 \pm 0.66$ \\
\hline BI dose at visit 3 (U/day/kg) & $0.21 \pm 0.09$ & $0.21 \pm 0.09$ \\
\hline Staple food at visit 3 (g) & $161.37 \pm 43.61$ & $154.81 \pm 41.17$ \\
\hline Vegetables at visit 3 (g) & $185.73 \pm 65.28$ & $180.62 \pm 63.87$ \\
\hline Fruit at visit $3(\mathrm{~g})$ & $101.23 \pm 41.63$ & $98.59 \pm 38.42$ \\
\hline Meat at visit $3(\mathrm{~g})$ & $104.63 \pm 36.96$ & $101.54 \pm 34.38$ \\
\hline $\begin{array}{l}\text { Days of participating in }>30 \text { min physical activity in past } 7 \\
\text { days at visit } 3\end{array}$ & $5.98 \pm 1.97$ & $5.83 \pm 2.10$ \\
\hline $\begin{array}{l}\text { Days of participating in specific exercise session in past } 7 \\
\text { days at visit } 3\end{array}$ & $4.17 \pm 2.88$ & $3.99 \pm 2.87$ \\
\hline \multicolumn{3}{|c|}{ Abbreviations: $\mathrm{BI}$, basal insulin; NPH, Neutral Protamine Hagedorn; OAD, oral antidiabetic drug. } \\
\hline \multicolumn{3}{|c|}{$\begin{array}{l}\text { All the listed factors at visit } 3 \text { were significantly different between two groups in full and sub- } \\
\text { populations. }\end{array}$} \\
\hline
\end{tabular}

Primary outcomes 
In the full population analysis, control rates of $\mathrm{HbA} 1 \mathrm{c} \leq 6.5 \%$ and $\mathrm{HbA} 1 \mathrm{c}<7.0 \%$ were both significantly higher in $\mathrm{BI}$ with metformin group $(25.0 \%$ and $39.9 \%)$ than those in $\mathrm{BI}$ without metformin group $(22.1 \%$ and $36.4 \%)(P=0.0024 ; P=0.0011)$ (Table 3). No significant differences were shown in terms of hypoglycemia between two groups at visit 3 . The statistical significance of primary outcomes between two groups in the sub-analysis was consistent with that in the full population analysis, with higher control rates of $\mathrm{HbA} 1 \mathrm{c} \leq 6.5 \%(30.1 \%$ vs. $25.1 \%, \mathrm{P}=0.0003)$ and $\mathrm{HbA} 1 \mathrm{c}<7.0 \%(47.9 \%$ vs. $41.9 \%, \mathrm{P}=0.0001)$ in $\mathrm{BI}$ with metformin group. (Table 3 ) 
Table 3

Effectiveness and safety of BI with or without metformin after 6 months - results of propensity score regression ${ }^{a}$

\section{Dependent variables}

The full population $(\mathrm{N}=12358)$

$\mathrm{HbA} 1 \mathrm{c}$ control, \% $(95 \% \mathrm{Cl})$

$\leq 6.5 \%$

$<7.0 \%$

HbA1c level (\%), mean $(95 \% \mathrm{Cl})$

Mean level at v3

Change from baseline

FPG control (< $7.0 \mathrm{mmol}), \%(95 \% \mathrm{Cl})$

FPG level (mmol/L), mean $(95 \% \mathrm{Cl})^{b}$

Mean level at v3

Change from baseline

Insulin dose $(\mathrm{U} /$ day $/ \mathrm{kg})$, mean $(95 \% \mathrm{Cl})$

Insulin dose at v3

Change from $v 1$ to $v 3$

Weight $(\mathrm{kg})$, mean $(95 \% \mathrm{Cl})$

Mean level at v3

Change from baseline

Hypoglycemia, \% (95\% Cl)

The sub-population $(\mathrm{N}=\mathbf{8 0 3 5})$

$\mathrm{HbA} 1 \mathrm{c}$ control, \% (95\% Cl)

$\leq 6.5 \%$

$<7.0 \%$

HbA1c level (\%), mean (95\% Cl)

Mean level at v3

Change from baseline

FPG control (<7.0 mmol), \% (95\% Cl)
BI with metformin

$N=6621$

$25.0(20.9,29.5)$

$39.9(345.1,45.0)$

$22.1(18.4,26.3)$

$36.4(31.7,41.3)$

0.0024

0.0011

$7.4(7.3,7.6)$

$7.5(7.4,7.6)$

0.0302

$-1.8(-1.9,-1.6)$

$-1.7(-1.9,-1.5)$

0.1839

$36.0(31.2,41.0)$

$33.5(28.9,38.5)$

0.0515

$8.3(8.1,8.5)$

$8.3(8.1,8.5)$

0.6875

$-2.9(-3.3,-2.5)$

$-3.0(-3.3,-2.5)$

0.5169

$0.33(0.32,0.33)$

$0.34(0.33,0.34)$

$<.0001$

$0.064(0.059,0.060)$

$0.068(0.063,0.070)$

0.0035
$70.1(69.3,70.0)$

$0.35(0.11,0.59)$

$11.2(9.1,13.8)$

$N=4269$

$30.1(28.4,31.8)$

$47.9(46.1,49.7)$

$25.1(23.4,26.8)$

0.0003

$41.9(39.9,43.8)$

0.0001
0.4862

0.7223

0.2965
$N=3799$

$7.2(7.2,7.3)$

$7.3(7.3,7.4)$

0.0009

$-2.0(-2.1,-1.9)$

$-1.9(-2.0,-1.9)$

0.4175

$49.6(47.5,51.8)$

$46.8(44.5,49.1)$

0.1319 


\begin{tabular}{|c|c|c|c|}
\hline Dependent variables & BI with metformin & Bl without metformin & $P$ value \\
\hline \multicolumn{4}{|c|}{ FPG level $(\mathrm{mmol} / \mathrm{L})$, mean $(95 \% \mathrm{Cl})^{\mathrm{c}}$} \\
\hline Mean level at v3 & $7.5(7.4,7.6)$ & $7.6(7.5,7.7)$ & 0.2212 \\
\hline Change from baseline & $-3.7(-3.8,-3.5)$ & $-3.7(-3.9,-3.5)$ & 0.8499 \\
\hline \multicolumn{4}{|c|}{ Insulin dose (U/day/kg), mean (95\% Cl) } \\
\hline Insulin dose at v3 & $0.21(0.20,0.21)$ & $0.22(0.21,0.21)$ & $<.0001$ \\
\hline Change from $v 1$ to $v 3$ & $0.033(0.031,0.035)$ & $0.037(0.035,0.039)$ & 0.0073 \\
\hline \multicolumn{4}{|l|}{ Weight $(\mathrm{kg})$, mean $(95 \% \mathrm{Cl})$} \\
\hline Mean level at v3 & $67.4(67.0,67.8)$ & $67.7(67.3,68.1)$ & 0.2965 \\
\hline Change from baseline & $-0.12(-0.22,-0.02)$ & $0.15(0.042,0.24)$ & 0.0013 \\
\hline Hypoglycemia, \% (95\% Cl) & $7.2(6.3,8.2)$ & $6.9(6.0,8.0)$ & 0.6892 \\
\hline \multicolumn{4}{|c|}{$\begin{array}{l}\text { a: Independent variables include propensity score, process confounding factors and metformin group. } \\
\text { Baseline covariates balanced by propensity score adjustments between two groups include regions, } \\
\text { hospital level, gender, age, current residence, medical insurance (actual proportion of out of pocket), } \\
\text { BMl, diabetes duration, OAD treatment duration, HbA1c level and FPG level at baseline, diabetes } \\
\text { complication, number and types of OADs before BI therapy, BI types at BI therapy initiation. Process } \\
\text { confounding factors controlled in the models include the number of OADs and total insulin dose } \\
\text { (uncontrolled in the model when analyzed as the outcome) at visit 3, physical activity and diet } \\
\text { consumption. }\end{array}$} \\
\hline \multicolumn{4}{|c|}{$\begin{array}{l}\text { b: } 4018 \text { patients had missing data for FPG level with } 2190 \text { patients from BI with metformin group and } \\
1828 \text { patients from BI without metformin group }\end{array}$} \\
\hline \multicolumn{4}{|c|}{$\begin{array}{l}\text { C: } 2440 \text { patients had missing data for FPG level with } 1306 \text { patients from BI with metformin group and } \\
1134 \text { patients from BI without metformin group }\end{array}$} \\
\hline \multicolumn{4}{|c|}{ Abbreviations: v1 (baseline, visit 1), v3 (6-month, visit 3) } \\
\hline
\end{tabular}

\section{Secondary outcomes}

In the full population analysis, BI with metformin group had lower adjusted mean of insulin dose at v3 (0.33 vs. $0.34 \mathrm{U} /$ day $/ \mathrm{kg}, \mathrm{P}<0.0001)$, lower increment of insulin dose from v1 to v3 (0.064 vs. 0.0068 $\mathrm{U} /$ day $/ \mathrm{kg}, \mathrm{P}=0.0035)$, and lower $\mathrm{HbA1c}$ mean level at v3 $(7.4 \%$ vs. $7.5 \%, \mathrm{P}=0.0302)$ compared with $\mathrm{BI}$ without metformin group. Weight, $\mathrm{HbA} 1 \mathrm{c}$ and FPG levels changes from $\mathrm{v} 1$ to $\mathrm{v} 3$ and adjusted mean levels at v3 of FPG and weight were not significantly different between the two groups, as well as FPG control rate $(<7.0 \mathrm{mmol} / \mathrm{L})$. (Table 3$)$

In the sub-analysis for patients remained at the same BI therapy consistently with or without metformin during 6-month follow-up, BI with metformin group also had a lower insulin dose at v3 (0.21 vs. 0.22 $\mathrm{U} / \mathrm{day} / \mathrm{kg}, \mathrm{P}<0.0001)$, less increment of insulin dose from $\mathrm{v} 1$ to $\mathrm{v} 3(0.0033 \mathrm{vs} .0 .0037 \mathrm{U} / \mathrm{day} / \mathrm{kg}, \mathrm{P}=$ $0.0073)$, and lower level of HbA1c $(7.3 \%$ vs. $7.2 \% \mathrm{P}=0.0009)$ compared with $\mathrm{BI}$ without metformin group. The sub-analysis showed BI with metformin group had slightly weight reduction while BI without 
metformin group had weight gain at v3 $(-0.12$ vs $0.15 P=0.0013)$. No significant differences were observed in mean levels of FPG and weight, FPG control rate $(<7.0 \mathrm{mmol} / \mathrm{L})$, as well as $\mathrm{HbA} 1 \mathrm{c}$ and FPG levels changes from v1 to v3 in the sub-analysis. (Table 3 )

\section{Discussion}

This real-world study observed that around half of T2DM patients initiating BI therapy due to uncontrolled hyperglycemia on OADs were prescribed concomitant with metformin. BI therapy with metformin showed higher rates of glycemic control, less increment of insulin dose and less weight gain compared with $\mathrm{BI}$ without metformin after 6-month follow-up. These results suggest that metformin-based BI therapy regimen can be advantageous for patients with suboptimal glycemic control on OADs in improving glycemic control and reducing weight gain.

In this real-world study, the overall proportion of patients using metformin prior to BI therapy was $65.2 \%$, in which $72.2 \%$ continued metformin after BI therapy. After BI therapy, the overall proportion of metformin use was $53.1 \%$. An observational study done in the US reported more than $70 \%$ patients taking metformin before insulin therapy and $80.3 \%$ patients continued it after insulin initiation. The proportion of metformin use after insulin therapy was also higher (63.8\%) in Pilla et al.'s study (23). Another study with commercial insurance data also reported $72.7 \%$ patients taking metformin before insulin initiation and metformin was continued in $84.6 \%$ patients after insulin therapy. (24) Given its low price, safety profile, and potential cardiovascular protection, metformin is recommended as first-line glucose lowering drug for treating T2DM unless contraindication or intolerance, which is also suggested being continued in combination with other agents including insulin $(9,10,25)$. Nevertheless, $35 \%$ of patients were not treated by metformin before BI therapy and more than one fourth of patients stopped metformin after initiating $\mathrm{BI}$ in our study, which may suggest the underuse of metformin in Chinese patients with T2DM in spite of possible contraindication or intolerance among some patients. One reason might be clinicians tending to stop metformin to avoid risks from complex treatment, especially for those with contraindication. Nevertheless, this study didn't collect information on patient's estimated glomerular filtration rate (eGFR) or side effects of metformin. Further studies about factors associated with metformin discontinuation after BI therapy are required.

In our study, the sub-analysis tends to provide higher estimates of the effectiveness and safety for BI with metformin over without metformin than the full population analysis. Patients in full population analysis who switched to other treatment regimens might be those with suboptimal glycemic control at the initial BI treatment. Therefore, compared with sub-analysis, the full population analysis containing patients that switched treatment regimen during follow-up may also dilute inter-group differences if a higher proportion of patients switching to other regimens in BI with metformin group remained having suboptimal glycemic control than those in BI without metformin group. In terms of safety, as patients could not tolerate prescribed regimen or experienced adverse events, they tend to not continue with the initial regimen. In this way, exclusion of these patients in the sub-analysis might make a study drug appear safer than it really is. However, given the wide acknowledged safety profile of metformin (12), risks of hypoglycemia or 
other adverse effects caused by BI with metformin should be low in our study. Despite of some discordances between the full population analysis and the sub-analysis, they both illustrated the advantages of metformin-based BI therapy in increasing glycemic control and insulin dose.

Most previous randomized studies reported the superiority of insulin combined with metformin in reducing $\mathrm{HbA} 1 \mathrm{c}$, weight gain, insulin dose or hypoglycemia compared with insulin alone or insulin plus other OADs, whereas they were not always concordant with each other $(14,16-18)$. In a study by Hollander et al., insulin plus metformin was reported to be associated with more $\mathrm{HbA} 1 \mathrm{c}$ reduction, less weight gain and lower hypoglycemia rates compared with insulin plus sulfonylurea (17). Nevertheless, in a study by Lund et al., insulin plus metformin showed less weight gain ( $2.22 \mathrm{vs.} 4.73 \mathrm{~kg}$ ) but equivalent $\mathrm{HbA} 1 \mathrm{c}$ reduction (from $8.15 \pm 1.32$ to $6.72 \pm 0.66 \%$ vs. $8.07 \pm 1.49$ to $6.90 \pm 0.66 \%$ ) compared with insulin plus repaglinide (26). Strowig et al. also reported lower weight gain ( $0.5 \mathrm{vs} .4 .4 \mathrm{~kg})$ and fewer hypoglycemic episodes (0.6 vs. 2.0/1.7 episodes/person/month) in insulin plus metformin group compared with insulin alone or insulin plus troglitazone but a comparable average reduction in $\mathrm{HbA} 1 \mathrm{c}$ with insulin plus metformin as with insulin alone (both from $8.8 \pm 1.2$ to $7.1 \pm 1.0 \%$ ) (16). However, previous randomized studies did not specify the type of insulin as basal insulin $(16,26)$ or the participants as insulin-naïve patients who initiated BI therapy due to suboptimal glucose control on OADs (17). Given the current guidelines consistently recommend timely initiating insulin therapy when glycemic target cannot be maintained by OADs in addition to lifestyle management, and that $\mathrm{BI}$ is considered as the most appropriate initial insulin regimen, $(9,10)$ this real-world study correspondingly investigated the effectiveness and safety of initiating BI plus metformin in Chinese T2DM patients of which the results can further support its application in China.

Strengths of this analysis are its large size, wide geographic variation and reliance on data from prospective, real-world clinical practice, which make the findings in our study more representative for a broader population of those patients and more capable of reflecting actual medication practice and its effectiveness and safety than in traditional randomized clinical trials. In addition, appropriate adjustments for various potential clinical and non-clinical factors improved the robustness of this statistical analysis.

One major limitation of this study was the possibility of confounding, which was one of the common flaws in observational studies. Although propensity score-adjusted regression model was applied to address confounding by covariates of baseline characteristics and factors at 6-month follow-up were also controlled, some differences between groups may be still present. Moreover, given its large size, the statistical difference may not necessarily conclude the clinical relevance. Another limitation is that data on eGFR reflecting renal function and gastrointestinal side effects were not collected. Finally, as this study only evaluated the outcomes of glycemic control, weight and hypoglycemia due to the short followup period, the impact on long-term outcomes such as microvascular or macrovascular complications and death were not assessed.

\section{Conclusions}


In conclusion, adopting basal insulin therapy with metformin in T2DM patients with previously suboptimal glycemia control by OADs was associated with better glycemic control and probably lower insulin dose and less weight gain compared with BI without metformin. However, the clinical relevance of these results must be interpreted with caution.

\section{Abbreviations}

$\mathrm{BI}$

Basal insulin

BMI

Body mass index

$\mathrm{Cl}$

Confidence intervals

DBP

Diastolic blood pressure

FPG

Fasting plasma glucose

$\mathrm{HbA1c}$

Glycated hemoglobin

IRB

Institutional Review Board

$\mathrm{NPH}$

Neutral Protamine Hagedorn.

ORBIT

Observational Registry for Basal Insulin Treatment

OAD

Oral antidiabetic drug

T2DM

Type 2 diabetes mellitus

SBP

Systolic blood pressure

SD

Standard deviation

\section{Declarations}

\section{Ethics approval and consent to participate}

The ORBIT protocol was approved by the Institutional Review Board (IRB) of Peking University and, when necessary, by local IRBs. Written informed consents were obtained from all patients. 


\section{Consent for publication}

Not applicable

\section{Availability of data and materials}

The data used to support the findings of this study are available from the corresponding author upon request.

\section{Competing interests}

L. J. reported receiving consulting and lecture fees from Eli Lilly, Bristol-Myers Squibb, Novartis, Novo Nordisk, Merck, Bayer, Takeda, Sanofi, Roche and Boehringer Ingelheim, and research grants from Roche and Sanofi. The other authors declare that they have no conflicts of interest.

\section{Funding}

This investigator sponsored study received funding from Sanofi.

\section{Authors' Contributions}

L.J and P.Z. contributed to the study design, conduct, and data interpretation. X.L. and J.J. contributed to the data analysis. H.Z., Y.L., D.Z., M.C. and D.W contributed to the interpretation of findings and drafting of the manuscript.

\section{Acknowledgements}

The authors would like to thank investigators from the secondary and tertiary hospitals for their hard work and patients for their participation, without whom this study would not have been possible.

\section{References}

1. Reusch JE, Manson JE. Management of Type 2 Diabetes in 2017: Getting to Goal. JAMA. 2017;317(10):1015-6.

2. Xu Y, Wang L, He J, Bi Y, Li M, Wang T, et al. Prevalence and control of diabetes in Chinese adults. JAMA. 2013;310(9):948-59.

3. Bolli GB, Lucidi P, Porcellati F, Fanelli CG. Pivotal role of timely basal insulin replacement after metformin failure in sustaining long-term blood glucose control at a target in type 2 diabetes. Diabetes Care. 2011;34(Suppl 2):220-4.

4. Donner T, Munoz M. Update on insulin therapy for type 2 diabetes. J Clin Endocrinol Metab. 2012;97(5):1405-13.

5. Inzucchi SE, Bergenstal RM, Buse JB, Diamant M, Ferrannini E, Nauck M, et al. Management of hyperglycemia in type 2 diabetes, 2015: a patient-centered approach: update to a position statement 
of the American Diabetes Association and the European Association for the Study of Diabetes. Diabetes Care. 2015;38(1):140-9.

6. Spollett GR. Insulin initiation in type 2 diabetes: what are the treatment regimen options and how can we best help patients feel empowered? J Am Acad Nurse Pract. 2012;24(Suppl 1):249-59.

7. Edelman S, Pettus J. Challenges associated with insulin therapy in type 2 diabetes mellitus. Am J Med. 2014;127(10 Suppl):11-6.

8. Nathan DM, Buse JB, Davidson MB, Ferrannini E, Holman RR, Sherwin R, et al. Medical management of hyperglycaemia in type 2 diabetes mellitus: a consensus algorithm for the initiation and adjustment of therapy: a consensus statement from the American Diabetes Association and the European Association for the Study of Diabetes. Diabetologia. 2009;52(1):17-30.

9. American Diabetes A. 8. Pharmacologic Approaches to Glycemic Treatment: Standards of Medical Care in Diabetes-2018. Diabetes Care. 2018;41(Suppl 1):73-85.

10. CDS. Chinese Guideline for Type 2 Diabetes. (2017). Chin Journal of Diabetes Mellitus. 2017 (in Chinese);10(1):2-67.

11. Vos RC, van Avendonk MJ, Jansen H, Goudswaard AN, van den Donk M, Gorter K, et al. Insulin monotherapy compared with the addition of oral glucose-lowering agents to insulin for people with type 2 diabetes already on insulin therapy and inadequate glycaemic control. Cochrane Database Syst Rev. 2016;9:CD006992.

12. Rojas LB, Gomes MB. Metformin: an old but still the best treatment for type 2 diabetes. Diabetol Metab Syndr. 2013;5(1):6.

13. Goudswaard AN, Furlong NJ, Rutten GE, Stolk RP, Valk GD. Insulin monotherapy versus combinations of insulin with oral hypoglycaemic agents in patients with type 2 diabetes mellitus. Cochrane Database Syst Rev. 2004(4):CD003418.

14. Hemmingsen B, Christensen LL, Wetterslev J, Vaag A, Gluud C, Lund SS, et al. Comparison of metformin and insulin versus insulin alone for type 2 diabetes: systematic review of randomised clinical trials with meta-analyses and trial sequential analyses. BMJ. 2012;344:e1771.

15. Holden SE, Jenkins-Jones S, Currie CJ. Association between Insulin Monotherapy versus Insulin plus Metformin and the Risk of All-Cause Mortality and Other Serious Outcomes: A Retrospective Cohort Study. PLoS One. 2016;11(5):e0153594.

16. Strowig SM, Aviles-Santa ML, Raskin P. Comparison of insulin monotherapy and combination therapy with insulin and metformin or insulin and troglitazone in type 2 diabetes. Diabetes Care. 2002;25(10):1691-8.

17. Hollander P, Sugimoto D, Vlajnic A, Kilo C. Combination therapy with insulin glargine plus metformin but not insulin glargine plus sulfonylurea provides similar glycemic control to triple oral combination therapy in patients with type 2 diabetes uncontrolled with dual oral agent therapy. J Diabetes Complications. 2015;29(8):1266-71.

18. Lundby-Christensen L, Tarnow L, Boesgaard TW, Lund SS, Wiinberg N, Perrild H, et al. Metformin versus placebo in combination with insulin analogues in patients with type 2 diabetes mellitus-the 
randomised, blinded Copenhagen Insulin and Metformin Therapy (CIMT) trial. BMJ Open. 2016;6(2):e008376.

19. Ji L, Zhang P, Weng J, Lu J, Guo X, Jia W, et al. Observational Registry of Basal Insulin Treatment (ORBIT) in Patients with Type 2 Diabetes Uncontrolled by Oral Hypoglycemic Agents in China-Study Design and Baseline Characteristics. Diabetes Technol Ther. 2015;17(10):735-44.

20. Seaquist ER, Anderson J, Childs B, Cryer P, Dagogo-Jack S, Fish L, et al. Hypoglycemia and diabetes: a report of a workgroup of the American Diabetes Association and the Endocrine Society. Diabetes Care. 2013;36(5):1384-95.

21. Eeren HV, Spreeuwenberg MD, Bartak A, de Rooij M, Busschbach JJ. Estimating subgroup effects using the propensity score method: a practical application in outcomes research. Med Care. 2015;53(4):366-73.

22. Vansteelandt S, Daniel RM. On regression adjustment for the propensity score. Stat Med. 2014;33(23):4053-72.

23. Pilla SJ, Dotimas JR, Maruthur NM, Clark JM, Yeh HC. Changes in metformin use and other antihyperglycemic therapies after insulin initiation in patients with type 2 diabetes. Diabetes Res Clin Pract. 2018;139:221-9.

24. Xu Y, Pilla SJ, Alexander GC, Murimi IB. Use of non-insulin diabetes medicines after insulin initiation: A retrospective cohort study. PLoS One. 2019;14(2):e0211820.

25. Holman RR, Paul SK, Bethel MA, Matthews DR, Neil HA. 10-year follow-up of intensive glucose control in type 2 diabetes. N Engl J Med. 2008;359(15):1577-89.

26. Lund SS, Tarnow L, Frandsen M, Nielsen BB, Hansen BV, Pedersen O, et al. Combining insulin with metformin or an insulin secretagogue in non-obese patients with type 2 diabetes: 12 month, randomised, double blind trial. BMJ. 2009;339:b4324.

\section{Figures}




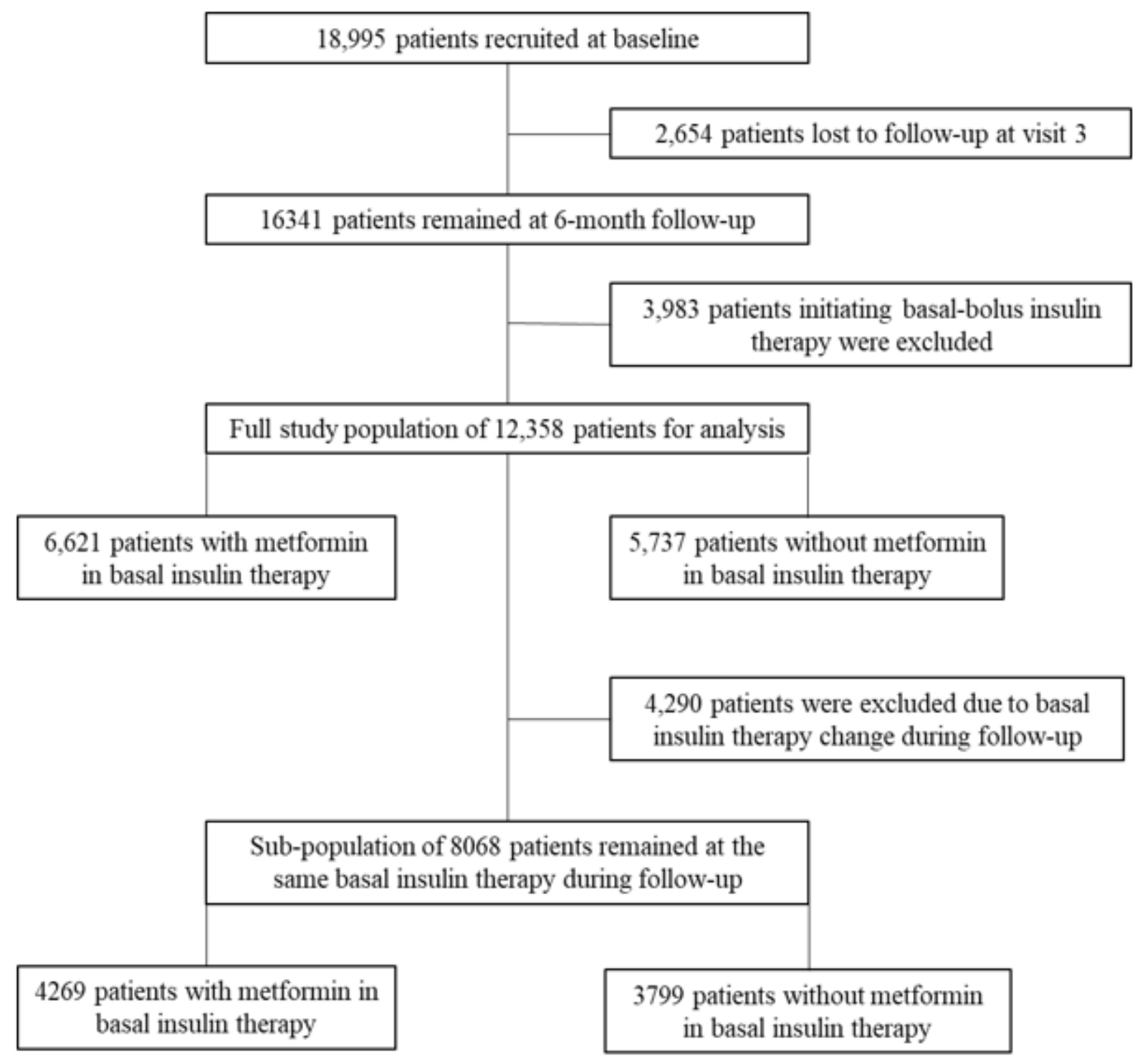

Figure 1

Flow of patients included in the analysis. 